\title{
Epidemiology of Hand Injury in Children
}

\begin{abstract}
Objective: To study the types and presentation of hand injuries in children. Method and Materials: A prospective study was done at the emergency department of Jinnah Postgraduate Medical Centre from 24th October, 2014 to 30th December, 2014 (a duration of six weeks). The data was collected on 50 out-patient children but we also followed the in-patient to find the outcome of their injuries. A self-designed questionnaire was administered and was filled by the parents of the participants. The questionnaire was explained in a one to one meeting by our volunteers. The volunteers were encouraging and friendly. Results: The most common reason of hand injury in our settings was due to road traffic accidents (34\%); followed by the second reason, a fall of an object on the hand. In most of the cases, $68 \%$ of the participants involved the right hand whereas in $32 \%$ of the participants, the left hand was involved. The most common bone to be fractured in children was the scaphoid with the 1st metacarpal bone. Conclusion: The goal of the study was to find out the various modes of presentation and types of the hand injuries in the accident and emergency department and to emphasize the regions commonly injured during a hand trauma. Better understanding of the type and manner of hand injuries will lead the physicians to emphasize on the areas more commonly injured and take suitable measures for their treatment accordingly. The rising number of hand injuries and knowledge about their pattern encourages the authorities to take suitable action and measures for its prevention and treatment which has been an area of neglect.
\end{abstract}

\section{Introduction}

Hand traumas in children are common, but often inadequately assessed and treated. (McCombe; 2015). They are prevalent in children of all ages and have been estimated to occur at an annual rate of 11.6 injuries per 1000 population. (Carrigan; 2014). The types of injuries vary with age, depending on the pattern of hand use (McCombe; 2015)

The hand is complicated in design and function; it is an amazing work of anatomic engineering and illustrates how form follows function in hands. Therefore, any injury to the underlying structure of the hand carries a potential for serious handicap. To reduce this risk, even the smallest hand injuries require proper medical evaluation (Chad; 2012). Lacerations have been estimated to account for $1 / 3$ of hand injuries presented to the emergency room (Carrigan; 2014). Metacarpal and phalanges fractures accounts for $21 \%$ of all pediatric fractures (Sivit; 2014). Although bones of adults and children share most of the same risk, the pattern of injury and accident are very different in adult life, (Grobbelaar; 1995) which is usually due to the presence of a growth plate in children.

When an accident occurs, the hand is the part of body most thrust out to lessen the consequences, resulting in a wide array of combinations of soft tissue and chondro-osseous injuries (Beatty; 1996). Radiography is the practicing way to diagnose these injuries but regardless of this, a large number of hand injuries are missed leading to delay of treatment, resulting in handicap as the children are incapable of giving a proper history. In this study, we reviewed the hand injuries in children presented to the emergency room so as to discuss the spectrum of its causes, types and the outcomes of these injuries. The purpose of this study was to emphasize this important area which has been a subject of neglect locally so as to come up with ideas to deal with this issue and increase its awareness.

\section{Method}

A prospective study was carried out at the emergency department of Jinnah Postgraduate Medical Centre in a period of six weeks from 24th October, 2014 to 30th December, 20114 after the approval of the research proposal by the Ethical Review Board. The information was collected from 50 outpatient children (all less than 12 years old) who were presented to the emergency department with hand injuries and were asked for a follow-up visit in two weeks.

The researcher filled a pre-designed questionnaire after getting informed consent from their parents, which includes the mode of injury, type of injury, and presentation in emergency. Inclusion criteria consisted of all children that should be completely vaccinated with no prior chronic illness and presented complaints of hand injury; we excluded all cases of old hand injuries. The results were computed by using the Statistical Package of Social Sciences version 20 (SPSS 20).

\section{Results}

The data was collected from $60 \%$ boys and $40 \%$ girls who were presented to the emergency room in duty hours. As shown in table 1 , the main cause of presentation was road traffic accidents $34 \%$ $(n=17)$ mostly reported in boys $(n=13)$ and falling of an object on their hand $32 \%(n=16)$ was common in girls $(n=9)$. The fractures were more common in right hand $68 \%$ than left hand $32 \%$ as shown in figure 1 . All the patients were conscious at the time of admission to emergency department. Of the total, $48 \%$ of the subjects reported dislocation of their wrist joint $(22 \%)$ common in girls and dislocation of metacarpal-phalangeal joint (22\%) common in boys as shown in table 2 .

Total $40 \%$ of the injuries involved damage to the ligaments and bone. The tendon was completely ruptured in $20 \%$ of the cases. The most common bone to be fractured was the Scaphoid along with the 1st Metacarpal Bone $14 \%$ followed by Scaphoid and Styloid alone $10 \%$ each. In $24 \%$ of the cases, only the base of metacarpals was fractured. In follow up, only $2 \%$ of the cases had amputation and only $2 \%$ of the cases reported sensory loss and weakness of the muscles of hand. 
Table 1

Relationship between gender and cause of injury Count

\begin{tabular}{|c|c|c|c|c|c|c|c|}
\hline & \multicolumn{6}{|c|}{ Cause of Injury } & \multirow[t]{2}{*}{ Total } \\
\hline & Penetrating & Blunt & Quarrel & Electric & $\begin{array}{c}\text { Road Traffic } \\
\text { Accident }\end{array}$ & Fall object on hand & \\
\hline \multirow{3}{*}{$\begin{array}{l}\text { Gender } \\
\mathrm{T}\end{array}$} & 2 & 2 & 3 & 3 & 13 & 7 & 30 \\
\hline & 1 & 1 & 3 & 2 & 4 & 9 & 20 \\
\hline & 3 & 3 & 6 & 5 & 17 & 16 & 50 \\
\hline
\end{tabular}

Figure 1

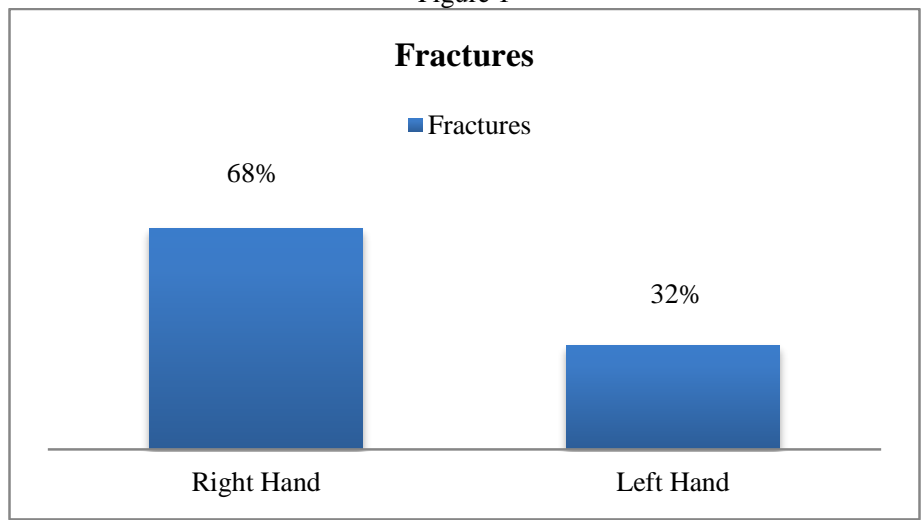

Table 2

Crosstab between gender and dislocation of joints Count

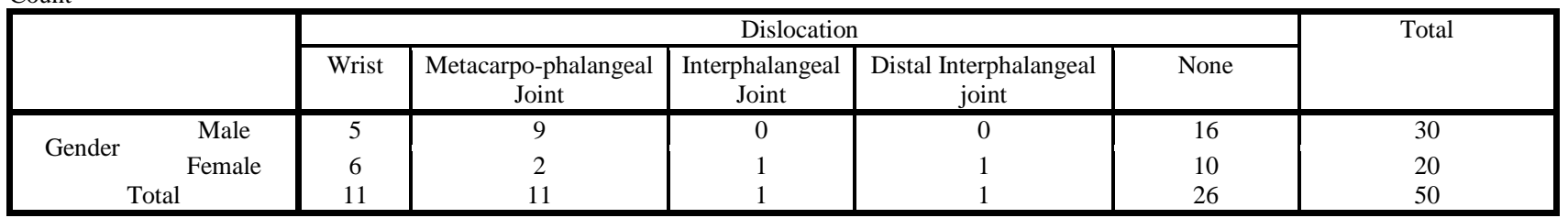

\section{Discussion}

Hand injuries are considered to be the most frequent body injuries, the treatment of which is of long duration and great community expense (Trybus; 2006). Amputation and complicated laceration were the most costly and resulted in more days lost from school (O'sullivan; 1993). The most frequent causes for admission were fractures (42\%), tendon lesions (29\%) and wounds (12\%), (Angermann; 1993) as consistent with our study. The dominant hand was more commonly injured although this was influenced by the cause of injury (Hill; 1998) similar to our findings in which $68 \%$ reported right hand fractures.

The pediatric bone is highly cellular and porous, and it contains a large amount of collagen and cartilage compared with adult bone. The tensile strength of the pediatric bone is less than that of the ligaments, so children are more likely to have bone injuries with mechanisms that would cause only ligamentous injuries in adults (Carson; 2006). A study reported that the fifth finger was the most commonly fractured digit (37\%), and the fifth metacarpal was the most commonly fractured bone $(\mathrm{P}<.01)$, (Bhende; 1993). This contrasts our study because we show that the Scaphoid along with the 1st Metacarpal Bone are the most common bones to be involved in fractures. The periosteum of the pediatric bone is comparatively more metabolically active than the adult periosteum leading to rapid and large callus formation, rapid union of fractures, and a higher potential for remodeling (Carson; 2006).
In keeping with the trend toward increasingly specialized surgical disciplines, the majority of pediatric hand injuries will be treated by surgeons with orthopedic, plastics, or hand surgery training. While most fractures heal uneventfully following closed treatment but several injuries require prompt diagnosis and specific treatment. Examining the injured hand can be challenging, especially in children. For this reason, the evaluation relies heavily on observation and passive tests. Radiographs must be specific for the parts of the hand that are injured. CT and MRI are rarely used but can be very useful (Cornwall; 2011). Almost all fractures healed in 2 to 3 weeks with an excellent functional outcome. Knowledge of epidemiology and etiology of hand fractures can serve as an essential first step in devising strategies to reduce the incidence of these hand injuries (Mahabir; 2001).

The purpose of the study was to increase community awareness. Equipment safety modifications are needed to eliminate this preventable problem (Lehrer; 1997) and understanding the patterns in which traumatic injuries occur in children is paramount to establishing effective injury prevention, as well as adapting treatment to optimize outcomes (Galano; 2005).

\section{Conflict of Interest}

There is no conflict of interest among the authors.

\section{Acknowledgment}

I would like to acknowledge Dr Tahir Kagazwala, Dr Muhammad Rehan Alamgir and Dr Ashba Mushtaqe for their immense support. 


\section{References:}

- Angermann, P., \& Lohmann, M. (1993). Injuries to the hand and wrist. A study of 50,272 injuries. Journal of Hand Surgery (British and European Volume), 18(5), 642-644.

- Bhende, M. S., Dandrea, L. A., \& Davis, H. W. (1993). Hand injuries in children presenting to a pediatric emergency department. Annals of emergency medicine, 22(10), 1519-1523.

- Beatty, E., Light, T. R., Belsole, R. J., \& Ogden, J. A. (1990). Wrist and hand skeletal injuries in children. Hand clinics, 6(4), 723-738.

- Carrigan, R. B. (2014). Extensor Tendon Injuries. In The Pediatric Upper Extremity (pp. 1-14). Springer New York.

- Chad Tarr MD, Hand Injuries, eMedicine Health, 5/11/2012, http://www.emedicinehealth.com/hand_injuries/article_em.html

- Carson, S., Woolridge, D. P., Colletti, J., \& Kilgore, K. (2006) Pediatric upper extremity injuries. Pediatric clinics of North America, 53(1), 41-67.

- Cornwall, R. (2011). Pediatric Hand Injuries. In Fundamentals of Pediatric Surgery (pp. 161-167). Springer New York.

- Grobbelaar, A. O., \& Hudson, D. A. (1995). Flexor pollicis longus tendon injuries in children. Annals of the Royal College of Surgeons of England, 77(2), 135.

- Galano, Gregory J., et al. "The most frequent traumatic orthopaedic injuries from a national pediatric inpatient population." Journal of Pediatric Orthopaedics 25.1 (2005): 3944.

- Hill, C., Riaz, M., Mozzam, A., \& Brennen, M. D. (1998). A regional audit of hand and wrist injuries a study of 4873 injuries. Journal of Hand Surgery (British and European Volume), 23(2), 196-200.

- Lehrer, M. S., Bozentka, D. J., Partington, M. T., Lee, B., \& Osterman, A. L. (1997). Pediatric hand injuries due to exercise bicycles. Journal of Trauma and Acute Care Surgery, 43(1), 100-102.

- Mahabir, R. C., kazemi, A. R., cannon, W. G., \& courtemanche, D. J. (2001). Pediatric hand fractures: a review. Pediatric emergency care, 17(3), 153-156.

- McCombe, D. (2015). Hand Injuries in Children. In Disorders of the Hand (pp. 285-309). Springer London.

- O'sullivan, M. E., \& Colville, J. (1993). The economic impact of hand injuries. Journal of Hand Surgery (British and European Volume), 18(3), 395-398.

- Sivit, A. P., DuPont, E. P., \& Sivit, C. J. (2014). Pediatric hand injuries: essentials you need to know. Emergency radiology, 21(2), 197-206.

- Trybus, M., Lorkowski, J., Brongel, L., \& Hl'adki, W. (2006). Causes and consequences of hand injuries. The American journal of surgery, 192(1), 52-57. 